\title{
A MATHEMATICAL MODEL FOR THE IMMUNOTHERAPY OF ADVANCED PROSTATE CANCER
}

\author{
TRAVIS PORTZ \\ Computer Sciences Department, University of Wisconsin-Madison \\ YANG KUANG \\ School of Mathematical and Statistical Sciences, Arizona State University
}

\begin{abstract}
A mathematical model of advanced prostate cancer treatment is developed to examine the combined effects of androgen deprivation therapy and immunotherapy. Androgen deprivation therapy has been the primary form of treatment for advanced prostate cancer for the past 50 years. While initially successful, this therapy eventually results in a relapse after two to three years in the form of androgen-independent prostate cancer. Intermittent androgen deprivation therapy attempts to prevent relapse by cycling the patient on and off treatment. Over the past decade, dendritic cell vaccines have been used in clinical studies for the immunotherapy of prostate cancer with some success. The model presented in this paper examines the efficacy of dendritic cell vaccines when used with continuous or intermittent androgen deprivation therapy schedules. Numerical simulations of the model suggest that immunotherapy can successfully stabilize the disease using both continuous and intermittent androgen deprivation.
\end{abstract}

\section{Introduction}

Prostate cancer is the most common type of cancer in American men and the second leading cause of cancer mortality ${ }^{11}$. Beginning as early as the second decade of life, the development of prostate cancer can require over 50 years to reach a detectable state ${ }^{1}$. Due to the slow growth rate of prostate cancer, chemotherapy has a very limited effect on the disease. Instead, treatment focuses on surgery and radiotherapy for localized disease and hormone therapy for metastatic cancer.

Since prostate cells and their malignant counterparts require stimulation by androgen to grow, advanced prostate cancer can be treated by androgen deprivation therapy (ADT). This therapy reduces the androgen-dependent (AD) cancer cells by preventing growth and inducing apoptosis ${ }^{1}$. While ADT is initially successful in most patients, almost all patients experience 
a relapse within several years. At this hormone refractory stage, the AD cells have been replaced by androgen-independent (AI) cells. These cells do not require the normal levels of androgen to sustain growth and are also resistant to the apoptotic effect of a low-androgen environment ${ }^{3}$.

Intermittent androgen deprivation (IAD) is an alternative therapy schedule where androgen deprivation therapy is administered until a patient experiences a remission of the disease and then is withheld until the disease progresses back to a certain level ${ }^{13}$. This therapy schedule aims to maintain apoptotic effect of androgen deprivation on the prostate cancer cells. Clinical studies have shown that patients are responsive to multiple cycles of the hormone therapy ${ }^{4,7,13}$. Progression to androgen independence was observed after two to five cycles lasting an average of 128 weeks ${ }^{7}$.

Additional treatment options are needed to prevent the progression to androgen independence and to treat those who already have androgen independent prostate cancer. Immunotherapy by dendritic vaccines is such an option. Dendritic cell (DC) vaccines are created by extracting DCs from a patient, loading the DCs with target antigens, and then reinfusing the DCs ${ }^{6}$. The antigen-loaded DCs activate naive $\mathrm{T}$ cells resulting in a cellular immune response against the target antigen. Clinical prostate cancer studies have used prostatic acid phosphatase (PAP) as the target antigen for prostate cancer DC vaccines ${ }^{6,19}$. These studies treated hormone refractory patients by administering DC vaccines loaded with PAP on a monthly basis. All patients developed an immune response following vaccination. Some patients experienced a significant decline in PSA level, and others experienced stabilization of their previously progressing disease.

Androgen deprivation therapy has been studied with a mathematical model to investigate the mechanism for androgen-independent relapse ${ }^{10}$. This model assumed continuous administration of androgen deprivation therapy and predicted that the treatment is only successful for a small range of biological parameters. Intermittent androgen deprivation was applied to this model and predicted that relapse can only be prevented by IAD if normal androgen levels have a negative effect on the growth rate of AI cells ${ }^{9}$. This is biologically unlikely since AI cells typically have androgen receptors with increased sensitivity ${ }^{8}$. Using biologically likely hypotheses for AI cell growth rates, mathematical models may or may not show that continuous therapy results in a longer time to androgen-independent relapse than intermittent therapy ${ }^{17,5}$.

Cell-mediated anti-tumor immune responses have been studied with a mathematical model by Kirschner and Panetta ${ }^{12}$. Their model examines 
the dynamics between the adaptive immune system, tumor cells, and the cytokine interleukin-2 (IL-2). The model shows the immune system can control tumors with average to high antigenicity at a dormant state. They also explore the possibility of treatment with IL-2. Their results suggest that treatment with IL-2 alone is not enough to clear the tumor without administering toxic levels of the cytokine.

In this paper, a mathematical model for advanced prostate cancer treatment is proposed which combines immunotherapy with intermittent androgen deprivation therapy. The immunotherapy portion of the model is based on the Kirschner and Panetta model, and the intermittent androgen deprivation therapy portion of the model is based on the work by Ideta et al. The model will investigate the efficacy of DC vaccines when administered before a hormone refractory stage of cancer. While the clinical studies found that the DC vaccines could slow the progression of the disease in hormone refractory patients, they did not show how the vaccine would affect patients actively undergoing hormone therapy. The model proposed in this paper will determine if intermittent therapy has any benefits over continuous therapy other than improved quality of life when combined with the use of DC vaccines. The necessary conditions for disease elimination or stabilization using this method of treatment will also be examined.

\section{The Model}

Prostate cancer treatment by immunotherapy and androgen deprivation therapy is modeled by a system of ordinary differential equations which takes the form

$$
\begin{aligned}
& \frac{d X_{1}}{d t}=\underbrace{r_{1}(A) X_{1}}_{\text {proliferation and death }}-\underbrace{m(A) X_{1}}_{\text {mutation to } \mathrm{AI}}-\underbrace{\frac{e_{1} X_{1} T}{g_{1}+X_{1}}}_{\text {killed by T cells }} \\
& \frac{d X_{2}}{d t}=\underbrace{r_{2} X_{2}}_{\text {proliferation and death }}+\underbrace{m(A) X_{1}}_{\text {mutation from AD }}-\underbrace{\frac{e_{1} X_{2} T}{g_{1}+X_{2}}}_{\text {killed by } \mathrm{T} \text { cells }} \\
& \frac{d T}{d t}=\underbrace{\frac{e_{2} D}{g_{2}+D}}_{\text {activation by dendritic cells }}-\underbrace{\mu T}_{\text {natural death }}+\underbrace{\frac{e_{3} T I_{L}}{g_{3}+I_{L}}}_{\text {clonal expansion }} \\
& \frac{d I_{L}}{d t}=\underbrace{\frac{e_{4} T\left(X_{1}+X_{2}\right)}{g_{4}+X 1+X 2}}_{\text {production by stimulated }}-\underbrace{\omega I_{L}}_{\text {clearance }}
\end{aligned}
$$




$$
\begin{aligned}
& \frac{d A}{d t}=\underbrace{\gamma\left(a_{0}-A\right)}_{\text {homeostasis }}-\underbrace{\gamma a_{0} u(t)}_{\text {deprivation therapy }} \\
& \frac{d D}{d t}=\underbrace{-c D}_{\text {natural death }} .
\end{aligned}
$$

Without the immunotherapy, the above model reduces to that of Ideta et $a l^{9}$. The variables used in the above model and their meanings are listed in Table 1. Parameter interpretations and estimates are given in Table 2.

Table 1.: Variables in the model

\begin{tabular}{cll}
\hline variable & meaning & unit \\
\hline \hline$X_{1}$ & number of androgen dependent cancer cells & cells \\
$X_{2}$ & number of androgen independent cancer cells & cells \\
$T$ & number of activated T cells & cells \\
$I_{L}$ & concentration of cytokines & $\mathrm{ng} / \mathrm{mL}$ \\
$A$ & concentration of androgen & $\mathrm{nmol} / \mathrm{mL}$ \\
$D$ & number of dendritic cells & cells \\
\hline
\end{tabular}

The androgen-dependent functions for AD cell growth and mutation are defined as follows:

$$
\begin{aligned}
& r_{1}(A)=\alpha_{1} \frac{A}{A+k_{1}}-\beta_{1}\left(k_{2}+\left(1-k_{2}\right) \frac{A}{A+k_{3}}\right) \\
& m(A)=m_{1}\left(1-\frac{A}{a_{0}}\right) .
\end{aligned}
$$

Notice that the net growth rate of $\mathrm{AD}$ cells is $-\beta_{1} k_{2}$ when $A=0$. The value of parameter $k_{2}$ is chosen such that $\beta_{1} k_{2}$ matches the rate of decline of serum PSA concentration during continuous androgen deprivation therapy 9 . The net growth rate of AI cells, $r_{2}$, is a constant in this version of the model. Ideta et al. proposed two alternatives for $r_{2}$ which assumed that androgen had a negative effect on the proliferation rate of AI cells ${ }^{9}$. Mutation from $\mathrm{AD}$ cells to AI cells occurs at a rate $m_{1}$ when $A=0$, and no mutation occurs when $A=a_{0}$. Larger values of $m_{1}$ result in a shorter time to androgen-independent relapse, thus relapse time can be used to estimate the value of $m_{1}{ }^{9}$.

Intermittent androgen deprivation therapy is modeled by (5) where $u(t)=1$ indicates an on-treatment period and $u(t)=0$ indicates an offtreatment period. During off-treatment periods, the androgen level tends toward homeostasis at the normal androgen level $a_{0}$. The androgen level 
Table 2.: Parameters in the model

\begin{tabular}{|c|c|c|c|}
\hline parameter & meaning & value & ref. \\
\hline$\alpha_{1}$ & AD cell proliferation rate & $0.025 /$ day & 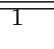 \\
\hline$\beta_{1}$ & AD cell death rate & $0.008 /$ day & 1 \\
\hline$k_{1}$ & $\begin{array}{l}\text { AD cell proliferation rate depen- } \\
\text { dence on androgen }\end{array}$ & $2 \mathrm{ng} / \mathrm{ml}$ & 9 \\
\hline$k_{2}$ & $\begin{array}{l}\text { effect of low androgen level on } \mathrm{AD} \\
\text { cell death rate }\end{array}$ & 8 & 2 \\
\hline$k_{3}$ & $\begin{array}{l}\text { AD cell death rate dependence on } \\
\text { androgen }\end{array}$ & $0.5 \mathrm{ng} / \mathrm{ml}$ & 9 \\
\hline$r_{2}$ & AI cell net growth rate & $0.006 /$ day & 1 \\
\hline$m_{1}$ & maximum mutation rate & $0.00005 /$ day & 9 \\
\hline$a_{0}$ & normal androgen concentration & $30 \mathrm{ng} / \mathrm{ml}$ & 9 \\
\hline$\gamma$ & $\begin{array}{l}\text { androgen clearance and production } \\
\text { rate }\end{array}$ & $0.08 /$ day & 9 \\
\hline$\omega$ & cytokine clearance rate & 10/day & 18 \\
\hline$\mu$ & $\mathrm{T}$ cell death rate & $0.03 /$ day & 12 \\
\hline$c$ & dendritic cell death rate & $0.14 /$ day & 15 \\
\hline$e_{1}$ & $\begin{array}{l}\text { maximum rate } \mathrm{T} \text { cells kill cancer } \\
\text { cells }\end{array}$ & $0-1 /$ day & 12 \\
\hline$g_{1}$ & $\begin{array}{l}\text { cancer cell saturation level for } \mathrm{T} \text { cell } \\
\text { kill rate }\end{array}$ & $10 \times 10^{9}$ cells & 12 \\
\hline$e_{2}$ & maximum $\mathrm{T}$ cell activation rate & $20 \times 10^{6}$ cells/day & 12 \\
\hline$g_{2}$ & $\begin{array}{l}\text { DC saturation level for } \mathrm{T} \text { cell acti- } \\
\text { vation }\end{array}$ & $400 \times 10^{6}$ cells & 19 \\
\hline$e_{3}$ & maximum rate of clonal expansion & $0.1245 /$ day & 12 \\
\hline$g_{3}$ & $\begin{array}{l}\text { IL-2 saturation level for } \mathrm{T} \text { cell } \\
\text { clonal expansion }\end{array}$ & $1000 \mathrm{ng} / \mathrm{ml}$ & 12 \\
\hline$e_{4}$ & maximum rate $\mathrm{T}$ cells produce $\mathrm{IL}-2$ & $\begin{array}{l}5 \times 10^{-6} \\
\mathrm{ng} / \mathrm{ml} / \text { cell/day }\end{array}$ & 12 \\
\hline$g_{4}$ & $\begin{array}{l}\text { cancer cell saturation level for } \mathrm{T} \text { cell } \\
\text { stimulation }\end{array}$ & $10 \times 10^{9}$ cells & 12 \\
\hline$D_{1}$ & $\mathrm{DC}$ vaccine dosage & $300 \times 10^{6}$ cells & 19 \\
\hline$c_{1}$ & AD cell PSA level correlation & $1 \times 10^{-9} \mathrm{ng} / \mathrm{ml} /$ cell & 9 \\
\hline$c_{2}$ & AI cell PSA level correlation & $1 \times 10^{-9} \mathrm{ng} / \mathrm{ml} /$ cell & 9 \\
\hline
\end{tabular}

decays at a rate $\gamma$ during on-treatment periods. The state variable $u(t)$ is 
controlled by monitoring the serum PSA level as follows:

$$
\begin{aligned}
& y(t)=c_{1} X_{1}+c_{2} X_{2} \\
& u(t)=\left\{\begin{array}{l}
0 \rightarrow 1 \quad \text { when } y(t)>L_{1} \text { and } \mathrm{d} y / \mathrm{d} t>0, \\
1 \rightarrow 0 \quad \text { when } y(t)<L_{0} \text { and } \mathrm{d} y / \mathrm{d} t<0,
\end{array}\right.
\end{aligned}
$$

where $y(t)$ is the serum PSA concentration. Androgen deprivation is switched on when the serum PSA concentration exceeds some level $L_{1}$ and switched off when the serum PSA concentration drops below some level $L_{0}$ with $L_{0}<L_{1}$. This method for IAD has been used in several clinical studies $4,7,13$.

Since $\mathrm{T}$ cells are activated and stimulated through interactions between proteins (antigens and cytokines) and receptors ${ }^{14}$, Michaelis-Menten kinetics are used for all immune response terms in the model. This is the approach taken by Kirschner and Panetta and is reasonable given that high levels of antigens and cytokines are likely to have a saturation effect on the T cells. IL-2 is included in the model to provide the clonal expansion dynamics of helper $\mathrm{T}$ cells. When stimulated by the antigens presented on tumor cells, the helper T cells produce IL-2. The IL-2 then stimulates the clonal expansion of $\mathrm{T}$ cells in a positive feedback loop ${ }^{14}$. The model assumes a constant ratio of cytotoxic and helper $\mathrm{T}$ cells, which greatly simplifies the model and should not have a significant impact on the long-term behavior of the system. The cytotoxic T cells interact with and kill the tumor cells based on antigen stimulation. The rate of interaction is assumed to be the same for both $\mathrm{AD}$ and $\mathrm{AI}$ cells. There is no biological reason to assume otherwise.

The antigen-loaded dendritic cells are modeled by (6) which assumes that the DCs undergo apoptosis at a constant rate and are not being replenished by any mechanisms other than further vaccinations. Vaccinations are administered every 30 days in model simulations. Each vaccination contains $D_{1}$ antigen-loaded DCs. The DCs are assumed to activate naïve T cells based on Michaelis-Menten kinetics as shown in (3). The model assumes that there are always naïve $\mathrm{T}$ cells available for activation.

The Michaelis-Menten terms could be replaced by simpler mass action terms to make the non-zero steady states easier to find analytically. However, the system is repeatedly being perturbed by the administration of DC vaccines, so steady-state analysis has limited use in this model. Since the model will be analyzed through numerical simulations, the more physically accurate Michaelis-Menten terms are used. 


\section{Numerical Simulations}

The mathematical model is simulated using the parameter estimates shown in Table 2. We vary the parameter $e_{1}$ based on the assumption that the $\mathrm{T}$ cell response against antigen-presenting tumor cells varies greatly from patient to patient. All simulations are run with the initial conditions $X_{1}(0)=14, X_{2}(0)=0.1, A(0)=30$, and $T(0)=I_{L}(0)=D(0)=0$ where cell populations are expressed in billions.

We first consider the case with androgen deprivation therapy only. The on-treatment PSA level $L_{1}$ is fixed at $15(\mathrm{ng} / \mathrm{ml})$, and the off-treatment PSA level $L_{0}$ is varied from 0 to $14(\mathrm{ng} / \mathrm{ml})$ to show the effects of IAD. The simulations in Figure 1 show that intermittent androgen therapy alone results in a shorter time to relapse than continuous therapy, correctly reproducing the results of the Ideta et al. model. Figure 2 shows the AD cell population being replaced by AI cells resulting in hormone refractory prostate cancer. With shorter treatment cycles, the androgen-independent relapse occurs sooner.

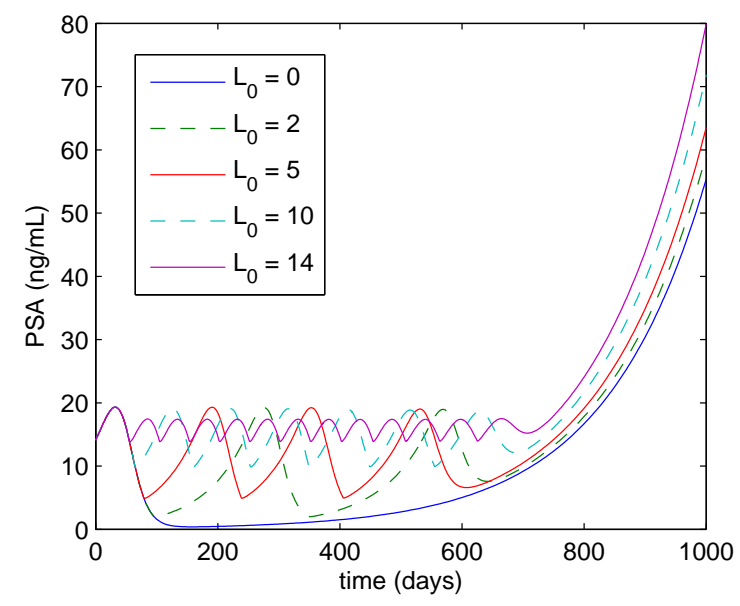

Figure 1.: Solutions of the model without immunotherapy. The on-treatment PSA level is fixed at $L_{1}=15$. When intermittent therapy is used (that is, when $L_{0}>0$ ), the cancer relapses sooner.

We next consider the case of continuous ADT with the $\mathrm{DC}$ vaccine. The parameter $e_{1}$ is varied to examine the effect that the immune response has on the tumor. The simulations in Figure 3 shows that the cancer 

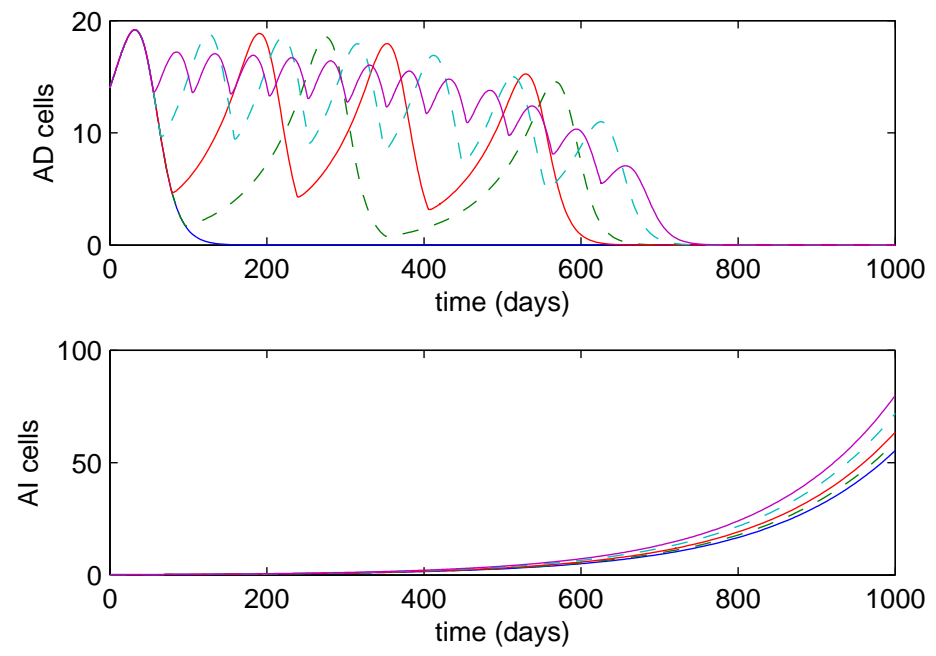

Figure 2.: AD and AI cell populations for the solutions in Figure 1.

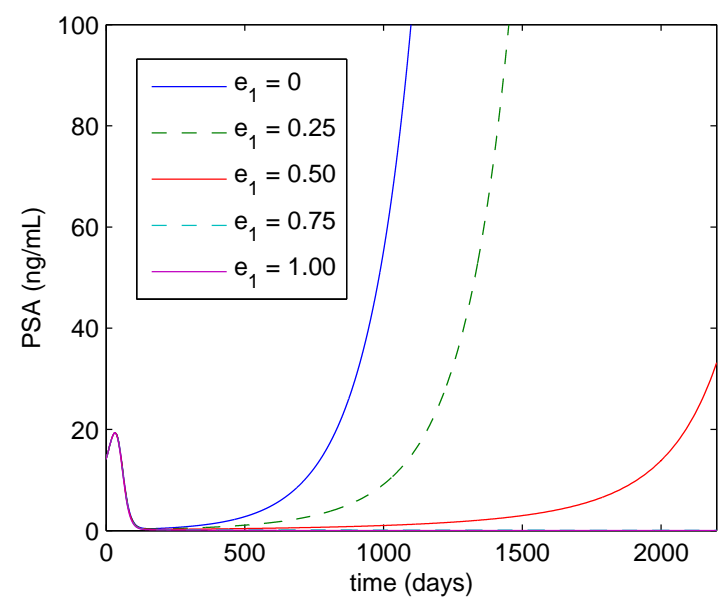

Figure 3.: Solutions of the model with continuous androgen deprivation therapy and immunotherapy. The cancer is eliminated with stronger antitumor immune responses, as seen for $e_{1}=0.75$ and $e_{1}=1.0$.

can be eliminated with a large enough value of $e_{1}$ within our parameter range. Figure 4 shows a log-lin plot of the AI cell population where we can see that the AI population grows exponentially for $e_{1} \leq 0.5$ and decays 

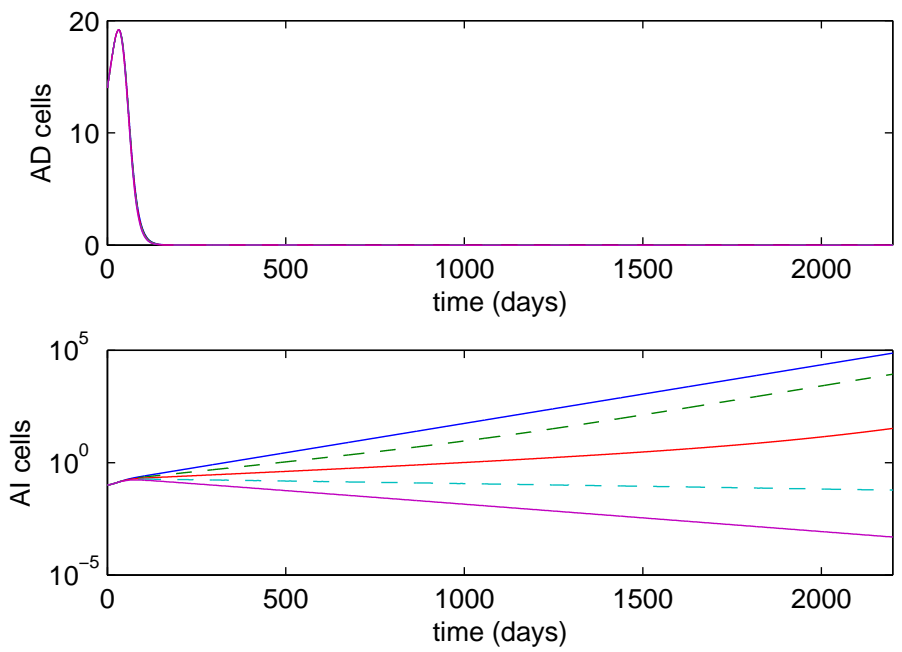

Figure 4.: AD and AI cell populations for the solutions in Figure 3.

exponentially for $e_{1} \geq 0.75$. The exact value of $e_{1}$ at which the cancer becomes manageable will be found in section 4 .

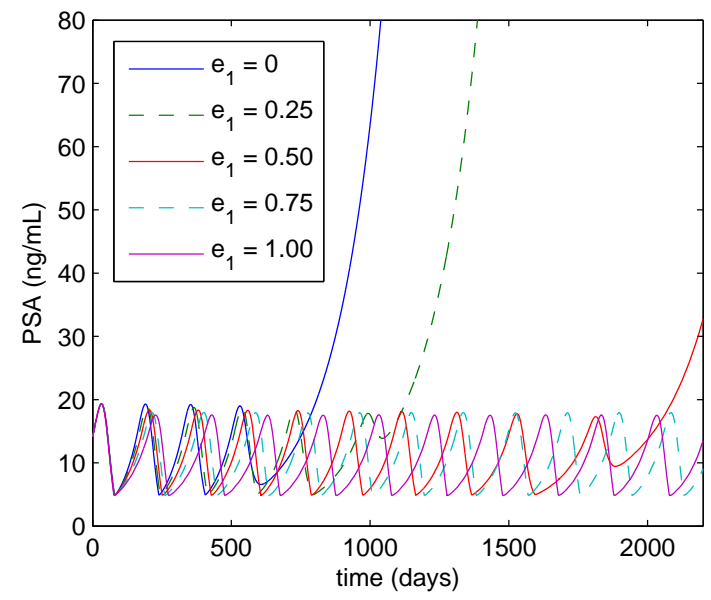

Figure 5.: Solutions of the model with intermittent androgen deprivation therapy and immunotherapy. The on-treatment PSA level is fixed at $L_{1}=$ 15. Relapse is prevented with stronger anti-tumor immune responses, as seen for $e_{1}=0.75$ and $e_{1}=1.0$. 

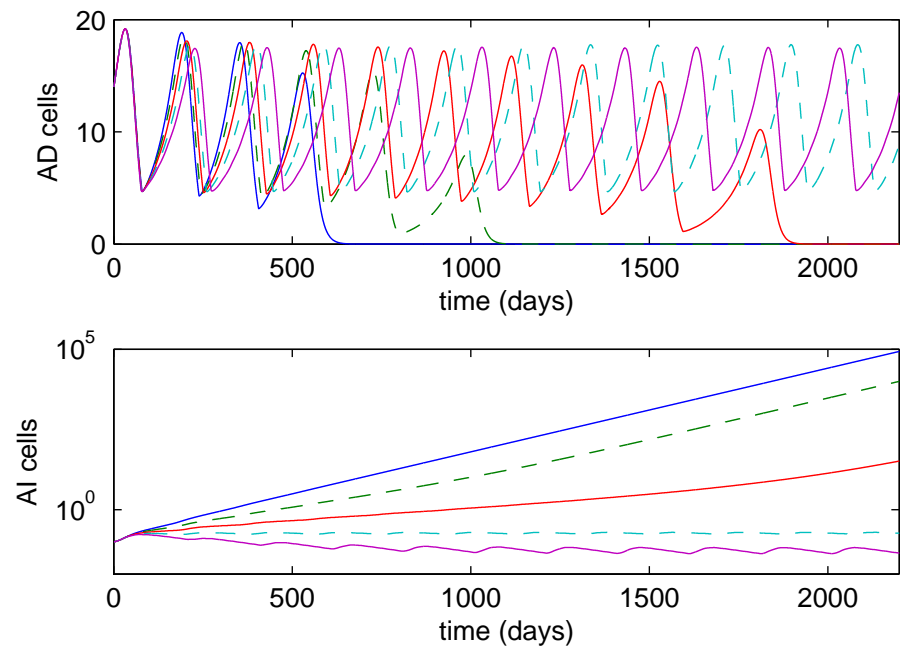

Figure 6.: AD and AI cell populations for the solutions in Figure 5.

Finally, we consider intermittent androgen deprivation therapy with the DC vaccine. Again, we vary the parameter $e_{1}$ to examine the effect of the immune response. The IAD is performed with $L_{0}$ fixed at $5(\mathrm{ng} / \mathrm{ml})$ and $L_{1}$ fixed at $15(\mathrm{ng} / \mathrm{ml})$. These values are similar to the PSA levels used for treatment scheduling in clinical studies ${ }^{4,7}$. The simulations in Figure 5 show that relapse is prevented for $e_{1} \geq 0.75$. Unlike continuous therapy, the intermittent therapy is not able to completely eliminate the disease. Due to the intermittent therapy schedule, the AD cell population will always oscillate between two sizes determined by $L_{0}$ and $L_{1}$ when androgen-independent relapse is prevented. The exact value of $e_{1}$ at which relapse becomes preventable will be found in section 4 .

\section{Mathematical Analysis}

Since the administration of DC vaccines will perturb the system away from any possible non-zero steady states, the only case where we could have a non-zero steady state is with a single administration of the DC vaccine at $t=0$ and continuous androgen deprivation therapy. Based on the model and also on the simulations in Figure 3 where DC vaccines are being administered every 30 days, we can see that there will be no non-zero steady state with just one vaccine administration of a reasonable dosage and with 


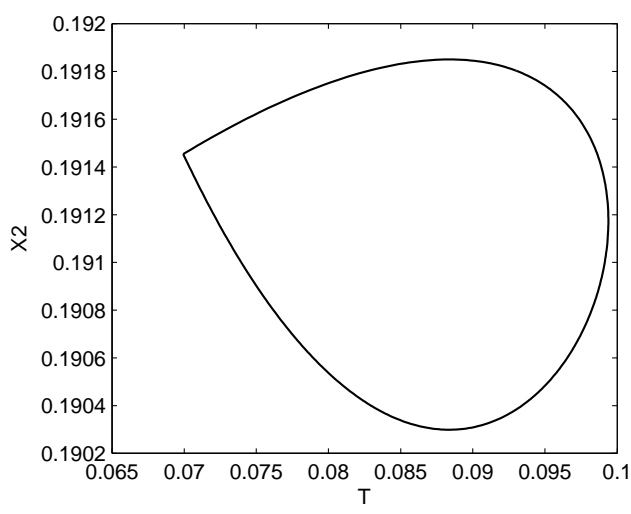

Figure 7.: The limit cycle found for $e_{1}=0.69197$ with continuous androgen deprivation therapy.

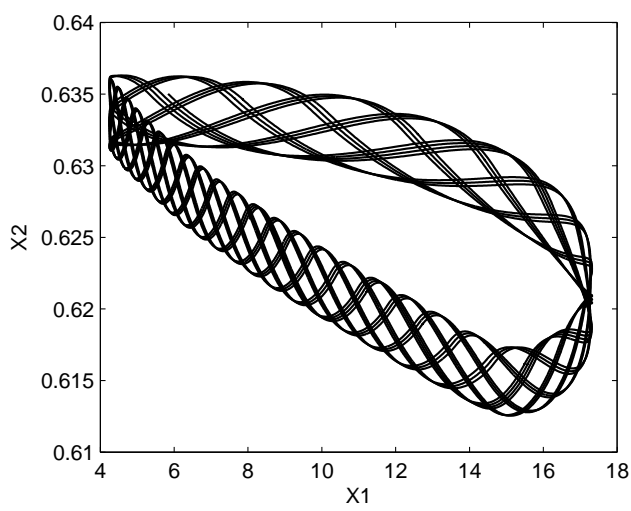

Figure 8.: The torus found for $e_{1}=0.68973$ with intermittent androgen deprivation therapy.

reasonable parameter estimates. For this reason, steady-state analysis will not be performed in this paper. Instead, we will find the values of $e_{1}$ at which the disease becomes controllable by immunotherapy for both the continuous and the intermittent therapy schedules.

We first find the value of $e_{1}$ at which continuous ADT combined with the $\mathrm{DC}$ vaccine is able to stabilize the disease. This value is known to be between 0.5 and 0.75 . The exact value can be found numerically by running simulations at parameter values within the known range and integrating the 


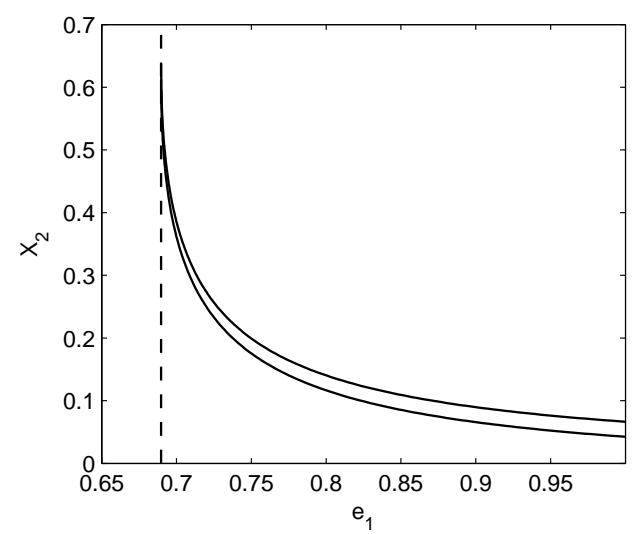

Figure 9.: A bifurcation diagram showing the maximum and minimum values of $X_{2}$ on the tori.

solution over a long period of time. When the solution is seen converging toward a limit cycle, the correct value of $e_{1}$ has been found. The limit cycle, shown in Figure 7 , was found at $e_{1} \approx 0.69197$. The period of this limit cycle is 30 days, the time between DC vaccinations. The cusp in the limit cycle is due to the discontinuity created by the repeated injections of DCs. For $e_{1}<0.69197$, the cancer eventually relapses and then grows exponentially as indicated by a diverging solution. For $e_{1}>0.69197$, relapse is prevented and the cancer decays exponentially.

We next find the value of $e_{1}$ at which IAD therapy combined with the $\mathrm{DC}$ vaccine is able to prevent an androgen-independent relapse. Again, we know this value is between 0.5 and 0.75 . With intermittent therapy, the solution will converges to a torus for a large enough value of $e_{1}$ rather than decay exponentially. Thus we need to find lowest value of $e_{1}$ at which the solution no longer diverges and grows exponentially. This is done again by integrating the solution over a long period of time and checking for divergence. The first stable torus, shown in Figure 8, was found at $e_{1} \approx$ 0.68973 . For larger values of $e_{1}$, the torus is located at lower values of $X_{2}$. The bifurcation diagram in Figure 9 shows the maximum and minimum values of $X_{2}$ on these tori. Tori are produced in this case because $X_{2}$ is experiencing oscillations due to the changes in T cell populations, which are periodically driven by the DC vaccinations, and the changes in the $X_{1}$ mutation rate, which oscillates due to the changing androgen levels. $X_{1}$ also experiences these oscillations, although the IAD therapy is the main 
source of the oscillations due to the much larger cell population and the effects of androgen on the growth rate of $\mathrm{AD}$ cells.

\section{Discussion}

The model has shown that androgen deprivation therapy alone is not enough to eliminate advanced prostate cancer or prevent an androgenindependent relapse, consistent with actual clinical results and the results of the Ideta et al. model in the case where the growth rate of AI cells is not inhibited by androgen. The model also shows that intermittent androgen deprivation therapy does not delay the progression of androgen-independent cancer when compared to continuous androgen deprivation. The model can help explain the shorter time to relapse. The number of mutations from $\mathrm{AI}$ to $\mathrm{AD}$ cells is highest early in the on-treatment period of therapy when androgen is low and the AD cell population is still relatively high. With continuous therapy, this high mutation rate only occurs once at the beginning of treatment, but with intermittent therapy, the high mutation rate occurs for each treatment cycle. This leads to a higher average mutation rate and therefore a higher average net growth rate for the AI cell population. While there is no benefit over continuous therapy with respect to relapse time, intermittent therapy is still an appealing treatment option due to the increased quality-of-life during the off-treatment periods.

When a dendritic cell vaccine is combined with continuous androgen deprivation, the model shows that the cancer can be eliminated with a relatively strong (but still within our reasonable parameter range) antitumor immune response. Clinical studies have shown that DC vaccines are able to stabilize disease progression in some patients with hormone refractory prostate cancer. Since these patients have androgen-independent cancer, we can safely assume that DC vaccines are capable of stopping the net growth of AI cells. When administered to a patient actively undergoing hormone therapy, we would then expect the DC vaccine to prevent an androgen-independent relapse while allowing continuous androgen deprivation therapy to eliminate the $\mathrm{AD}$ cell population. Thus the results of our model are reasonable in the case of DC vaccination combined with continuous androgen deprivation.

An interesting result of the model is that the DC vaccine is able to prevent relapse with a slightly weaker anti-tumor immune response when intermittent androgen deprivation is used instead of continuous androgen deprivation. While the difference was only small $\left(e_{1}=0.68973\right.$ compared 
to $e_{1}=0.69197$ ), the result was still surprising considering the effect that intermittent therapy has on relapse time without immunotherapy. This small difference can likely be attributed to the ability of the immune response to offset the higher mutation rate when intermittent therapy is used and also to the consistently larger cancer cell population which is necessary to stimulate the clonal expansion of $\mathrm{T}$ cells. With the low toxicity of DC vaccines ${ }^{6,19}$ and the quality-of-life benefits of IAD, the combination of these two treatments could be very advantageous over continuous ADT for the treatment of advanced prostate cancer.

The complexity of the model combined with its discrete nature makes standard analysis difficult. This forced the mathematical analysis in section 4 to use specific parameter values when finding the desired values of $e_{1}$, thus preventing us from having an algebraic expression for those values of $e_{1}$. Simplifying the model by replacing the Michaelis-Menten terms with mass action terms was considered in section 2, but the discrete nature of the model would still present difficulties for bifurcation analysis. Simplifying the model further may be possible by using continuous functions for the $D(t)$ and $u(t)$, although this would make clinical application of the model difficult.

Accuracy in the estimation of parameter values for the immune response is a significant limitation of the model. Most of the parameter estimates were based on the parameter estimates used in the Kirschner and Panetta model. While many of these parameter estimates were based on data from biological studies, the estimates are for generic anti-tumor immune responses. The proper parameter values for an immune response against prostate cancer may be significantly different, and additional data from clinical studies of DC vaccines is needed before these parameter estimates can be made accurately.

A possibly greater limitation of the model is the lack of regulatory $\mathrm{T}$ cells in the model. These cells are responsible for reducing sensitivity to self-antigens and preventing toxic levels of lymphocytes in response to a pathogen ${ }^{16}$. Since targeting cancer cells requires targeting a self-antigen, the regulatory $\mathrm{T}$ cells may have a significant suppressive effect on antitumor immune responses ${ }^{20}$. Several studies have examined ways to target regulatory $\mathrm{T}$ cells in order reduce their suppressive effect on immunotherapy 20. Including regulatory $\mathrm{T}$ cells in the model is a possible direction for future work, and the therapies which target the regulatory $\mathrm{T}$ cells could then be examined as well. 


\section{Acknowledgements}

This work is supported in part by NSF DMS-0436341 and DMS-0920744.

\section{References}

1. R.R. Berges, J. Vukanovic, J.I. Epstein, M. CarMichel, L. Cisek, D.E. Johnson, R.W. Veltri, P.C. Walsh, and J.T. Isaacs. Implication of cell kinetic changes during the progression of human prostatic cancer. Clin Cancer Res, 1(5):473-480, 1995.

2. N. Bruchovsky, L. Klotz, J. Crook, and S.L. Goldenberg. Locally advanced prostate cancer-biochemical results from a prospective phase II study of intermittent androgen suppression for men with evidence of prostate-specific antigen recurrence after radiotherapy. Cancer, 109(5), 2007.

3. N. Craft, C. Chhor, C. Tran, A. Belldegrun, J. DeKernion, O.N. Witte, J. Said, R.E. Reiter, and C. L. Sawyers. Evidence for Clonal Outgrowth of Androgen-independent Prostate Cancer Cells from Androgen-dependent Tumors through a Two-Step Process. Cancer Res, 59(19):5030-5036, 1999.

4. J.M. Crook, E. Szumacher, S. Malone, S. Huan, and R. Segal. Intermittent androgen suppression in the management of prostate cancer. Urology, 53(3):530-534, 1999.

5. S. Eikenberry, J. D Nagy, and Y. Kuang. The evolutionary impact of androgen levels on prostate cancer in a multi-scale mathematical model . Biology Direct, 5(24):1-28, 2010.

6. L. Fong, D. Brockstedt, C. Benike, J.K. Breen, G. Strang, C.L. Ruegg, and E.G. Engleman. Dendritic Cell-Based Xenoantigen Vaccination for Prostate Cancer Immunotherapy. J Immunol, 167(12):7150-7156, 2001.

7. S.L. Goldenberg, N. Bruchovsky, M.E. Gleave, L.D. Sullivan, and K. Akakura. Intermittent androgen suppression in the treatment of prostate cancer: a preliminary report. Urology, 45(5):839, 1995.

8. M.E. Grossmann, H. Huang, and D.J. Tindall. Androgen receptor signaling in androgen-refractory prostate cancer. J Nat Cancer Inst, 93(22):1687-1697, 2001.

9. A.M. Ideta, G. Tanaka, T. Takeuchi, and K. Aihara. A Mathematical Model of Intermittent Androgen Suppression for Prostate Cancer. J Nonlinear Sci, 18(6):593-614, 2008.

10. T.L. Jackson. A mathematical model of prostate tumor growth and androgenindependent relapse. Discrete Cont Dyn Syst Ser B, 4(1):187-202, 2004.

11. A. Jemal, R. Siegel, E. Ward, Y. Hao, J. Xu, T. Murray, and M.J. Thun. Cancer statistics, 2008. CA Cancer J Clin, 58(2):71-96, 2008.

12. D. Kirschner and J.C. Panetta. Modeling immunotherapy of the tumorimmune interaction. J Math Biol, 37(3):235-252, 1998.

13. L.H. Klotz, H.W. Herr, M.J. Morse, and W.F. Whitmore Jr. Intermittent endocrine therapy for advanced prostate cancer. Cancer, 58(11), 1986.

14. A. Lanzavecchia and F. Sallusto. Regulation of $\mathrm{T}$ cell immunity by dendritic cells. Cell, 106(3):263-266, 2001. 
15. M.T. Lotze and A.W. Thomson. Dendritic cells: biology and clinical applications. Academic Pr, 2001.

16. A. O'Garra and P. Vieira. Regulatory T cells and mechanisms of immune system control. Nature Med, 10(8):801-805, 2004.

17. T. Portz, Y. Kuang, and J. D. Nagy. A clinical data validated mathematical model of prostate cancer growth under intermittent androgen suppression therapy . AIP Advances, 2(011002):doi: 10.1063/1.3697848, 2012.

18. S.A. Rosenberg and M.T. Lotze. Cancer immunotherapy using interleukin-2 and interleukin-2-activated lymphocytes. Annu Rev Immunol, 4(1):681-709, 1986.

19. E.J. Small, P. Fratesi, D.M. Reese, G. Strang, R. Laus, M.V. Peshwa, and F.H. Valone. Immunotherapy of hormone-refractory prostate cancer with antigen-loaded dendritic cells. J Clin Oncol, 18(23):3894, 2000.

20. W. Zou. Regulatory T cells, tumour immunity and immunotherapy. Nature Rev Immunol, 6(4):295-307, 2006. 